\title{
Frequency and Sensitivity of Extended Spectrum Beta-Lactamase Positive Organisms in a Secondary and Tertiary Level Hospital Network in Dhaka
}

\author{
Shah Md Zahurul Haque Asna ${ }^{1}$, Shameem Akhter ${ }^{2}$, M Mushfequr Rahman $^{3}$, \\ Najib Mohammad ${ }^{4}$, M A Hafez $^{5}$ \\ Received: November 29, 2014 Accepted: April 12, 2015 \\ doi: http://dx.doi.org/10.3329/jemc.v5i2.23377
}

\begin{abstract}
Background: Extended spectrum $\beta$-lactamase (ESBL) positive organisms are now a global health concern including in Bangladesh. These are associated with treatment failure, increased morbidity and mortality and increased health care costs. In this study, frequency of ESBL positive organisms in some health care centres in Dhaka city has been observed and their current status of antibiogram has also been observed. Objective: To observe the current status of antibiogram of ESBL positive organisms. Materials and Methods: This cross-sectional study was done in the Department of Microbiology, Bangladesh Institute of Health Sciences (BIHS) General Hospital, Dhaka, Bangladesh from March, 2012 to February, 2013. Only E. coli and Klebsiella spp. from pus and urine specimens were included in this study. Isolation, identification and antibiotic sensitivity of the organisms were done by standard procedures. Results: Organisms (Escherichia coli and Klebsiella spp.) isolated from urine and pus collected from different sites of 472 subjects were studied. Predominant organisms were Escherichia coli (82.8\%) and remaining $17.2 \%$ were Klebsiella spp. ESBL positive organisms were higher in Escherichia coli (54.5\%) than in Klebsiella spp. (44.4\%) and higher in pus (77.0\%) than in urine (49.1\%) isolates. Imipenem is the most effective drug for treating ESBL positive organisms followed by colistin, tigecycline and piperacillin/tazobactam. Conclusion: Imipenem, colistin, tigecycline and piperacillin/tazobactam drugs should be kept reserved and used only when other effective drugs are not available so that emergence of resistance against these drugs is deferred. While reporting the culture and sensitivity tests, the ESBL positive organisms should be pointed out with comment like this - "The organisms are ESBL positive and resistant to penicillins, cephalosporins and monobactams".
\end{abstract}

Key words: ESBL; Drug resistance; Cephalosporins

J Enam Med Col 2015; 5(2): 80-87

\section{Introduction}

Extended spectrum $\beta$-lactamase (ESBL) producing organisms are now a global threat ${ }^{1}$ and its treatment has become difficult because of their drug resistance over a wide spectrum of $\beta$-lactam and non- $\beta$-lactam drugs.
These are emerging worldwide in community as well as in health care settings specially in Intensive Care Units (ICU). ${ }^{2}$ The ESBL isolates were first reported from Germany and England in $1983^{3,4}$ and subsequently in

1. Professor, Department of Microbiology, Bangladesh University of Health Sciences (BUHS), Dhaka

2. Associate Professor, Department of Microbiology, Bangladesh University of Health Sciences (BUHS), Dhaka

3. Assistant Professor, Department of Microbiology, Bangladesh University of Health Sciences (BUHS), Dhaka

4. Associate Professor, Department of Medicine, Bangladesh Institute of Health Sciences (BIHS) General Hospital, Dhaka

5. Professor, Department of Epidemiology \& Biostatistics, Bangladesh University of Health Sciences (BUHS), Dhaka

Correspondence Shah Md Zahurul Haque Asna, Email: asnabd04@yahoo.com 
USA in late 1988. ${ }^{4,5}$ In Bangladesh, it was reported first in 2001. ${ }^{6}$ ESBLs are enzymes that mediate resistance to $\beta$-lactam antibiotics, viz., penicillins, cephalosporins and monobactams, but do not affect cephamycins or carbapenems. Because of greatly extended substrate range, these enzymes are called extended spectrum $\beta$-lactamases. ${ }^{2}$ ESBL enzymes are most commonly produced by Escherichia coli (E. coli) and Klebsiella spp. and to some extent by other members of Enterobacteriaceae and Pseudomonadaceae. ${ }^{2,7}$

ESBL organisms have continued to increase in varieties (more than 400 variants detected) ${ }^{8}$ and prevalence and are now a global health concern. ${ }^{4,9,10}$ The ESBL organisms have implications for clinicians and patients because these are associated with treatment failure, increased morbidity and mortality, poor outcomes, increased length of stay (LOS) in hospital and health care costs. ${ }^{9}$ So, it is important to treat it properly as soon as it is diagnosed. Aim of this study was to observe the current status of antibiogram of ESBL positive organisms and their prevalence in and around Dhaka city.

\section{Materials and Methods}

This cross-sectional study was done in the Department of Microbiology, Bangladesh Institute of Health Sciences (BIHS) General Hospital, Dhaka, Bangladesh from March, 2012 to February, 2013. Specimens were collected from inpatient and outpateint departments of BIHS General Hospital and nine more hospitals/centres of Dhaka city affiliated to BIHS General Hospital.

Organisms isolated from urine and pus of 472 subjects were studied. There were 411 urine and 61 pus specimens. Pus was collected from diabetic foot lesions, post surgical infected wounds and traumatic wounds. Only E. coli and Klebsiella spp. were included in this study. Isolation, identification and antibiotic sensitivity of the organisms were done by standard procedures. ${ }^{11-14}$ For detection of ESBL positive organisms, screening test ${ }^{15}$ was done along with routine sensitivity test. Positive screening tests were confirmed later by Phenotypic Confirmatory Test (PCT) ${ }^{13}$

\section{Screening test}

Disks of cefotaxime (CTX), ceftazidime (CAZ) and amoxycillin/clavulanic acid (AMC) were placed in a line, placing $\mathrm{AMC}$ in the centre and other two on either side about $30 \mathrm{~mm}$ apart from centre to centre. Widening of cephalosporin's inhibition zone adjacent to the disk containing AMC was regarded as ESBL positive. ${ }^{14,15}$,

\section{Phenotypic confirmtory test (PCT)}

In this method, two sets were used. One set consisted of ceftazidime $(30 \mu \mathrm{g})$ disk and ceftazidime/clavulanic acid $(30 / 10 \mu \mathrm{g})$ disk. Another set consisted of cefotaxime $(30 \mu \mathrm{g})$ disk and cefotaxime/clavulanic acid $(30 / 10 \mu \mathrm{g})$. The disks among the sets were placed at least $30 \mathrm{~mm}$ apart (centre to centre). When the zone of inhibition by combination disks (ceftazidime/clavulanic acid or cefotaxime/clavulanic acid) was $\geqslant 5 \mathrm{~mm}$ than the zone of inhibition produced by ceftazidime/cefotaxime alone, the test was taken as PCT positive. ${ }^{13}$

\section{Media for sensitivity \\ Muller Hinton agar (Oxoid) ${ }^{13,14}$}

\section{Antibiotic disks used}

All the isolates were tested for sensitivity to amoxycillin/clavulanic acid $(20 / 10 \mu \mathrm{g})$, cefotaxime (30 $\mu \mathrm{g})$, ceftazidime $(30 \mu \mathrm{g})$, cefradine $(30 \mu \mathrm{g})$, cefoxitin $(30 \mu \mathrm{g})$, ampicillin $(10 \mu \mathrm{g})$, amikacin $(30 \mu \mathrm{g})$, imipenem $(10 \mu \mathrm{g})$, gentamicin $(10 \mu \mathrm{g})$, ciprofloxacin $(5 \mu \mathrm{g})$, nalidixic acid $(30 \mu \mathrm{g})$, nitrofurantoin $(300 \mu \mathrm{g})$, doxycycline $(30 \mu \mathrm{g})$, cotrimoxazole $(1.25 / 23.75 \mu \mathrm{g})$, colistin $(10 \mu \mathrm{g})$, tigecycline $(15 \mu \mathrm{g})$ and piperacillin/ tazobactam $(100 \mu \mathrm{g} / 10 \mu \mathrm{g}) .{ }^{13}$

The above mentioned antibiotic discs were placed on Muller Hinton agar according to standard procedure. ${ }^{14}$

\section{Results}

Isolation of ESBL positive organisms in BIHS General Hospital and different hospitals/centres located at different sites of Dhaka city has been shown in Table I. The highest isolation rate was in Wari Hospital (91.3\%) followed by BIHS General Hospital IPD, Mirpur (81.4\%) and Foot Care Hospital at Rampura (74.4\%). 
Table I: Distribution of ESBL positive organisms in different sites of Dhaka city

\begin{tabular}{|l|c|c|c|c|}
\hline \multicolumn{1}{|c|}{ Centre names } & \multicolumn{2}{|c|}{ Esch. coli } & \multicolumn{2}{|c|}{ Klebsiella } \\
\hline \multicolumn{1}{|c|}{ Isolated } & $\begin{array}{c}\text { ESBL+ve } \\
\text { Number (\%) }\end{array}$ & Isolated & $\begin{array}{c}\text { ESBL+ve } \\
\text { Number (\%) }\end{array}$ \\
\hline BIHSGH OPD, Mirpur & 148 & $77(52.0)$ & 23 & $04(17.4)$ \\
\hline BIHSGH IPD, Mirpur & 33 & $26(78.8)$ & 10 & $09(90.0)$ \\
\hline Savar & 41 & $18(43.9)$ & 04 & $03(75.0)$ \\
\hline Uttara & 32 & $15(46.9)$ & 07 & $01(14.3)$ \\
\hline Banani & 27 & $08(29.6)$ & 07 & $03(42.9)$ \\
\hline Bashaboo & 25 & $10(40.0)$ & 04 & $02(50.0)$ \\
\hline Jurain & 18 & $09(50.0)$ & 01 & $00(00)$ \\
\hline Dhanmondi Hospital & 20 & $10(50.0)$ & 06 & $01(16.7)$ \\
\hline Wari Hospital & 23 & $21(91.3)$ & 00 & $00(00)$ \\
\hline $\begin{array}{l}\text { Foot Care Hospital, } \\
\text { Rampura }\end{array}$ & 24 & $19(79.2)$ & 19 & $13(68.4)$ \\
\hline Total & 391 & $213(54.5)$ & 81 & $36(44.4)$ \\
\hline
\end{tabular}

BIHSGH, Bangladesh Institute of Health Sciences General Hospital

Frequency of organisms in different specimens is shown in Table II. E. coli was found more prevalent than Klebsiella spp. In case of E. coli $54.5 \%$ were ESBL positive and in case of Klebsiella $44.4 \%$ were ESBL positive. Frequency of ESBL positive organisms was more in case of pus $(77.0 \%)$ than in case of urine $(49.1 \%)$.

Table II: Frequency of ESBL positive Esch. coli and Klebseilla spp. in different specimens $(\mathrm{N}=472)$

\begin{tabular}{|l|c|c|c|c|c|c|}
\hline & \multicolumn{2}{|c|}{ Urine } & \multicolumn{2}{|c|}{ Pus } & \multicolumn{2}{c|}{ Total } \\
\hline Organisms & Isolates & ESBL+ve & Isolates & ESBL+ve & Isolates & ESBL+ve \\
& & Number (\%) & & Number (\%) & & Number (\%) \\
\hline Esch. coli & 354 & $183(51.7)$ & 37 & $30(81.1)$ & 391 & $213(54.5)$ \\
\hline Klebsiella spp. & 57 & $19(33.3)$ & 24 & $17(70.8)$ & 81 & $36(44.4)$ \\
\hline Total & 411 & $202(49.1)$ & 61 & $47(77.0)$ & 472 & $249(52.8)$ \\
\hline
\end{tabular}

In Table III sensitivity pattern of ESBL positive and ESBL negative E. coli and Klebsiella spp. to different antibiotics has been shown. In case of E. coli, all (100\%) of ESBL positive and ESBL negative strains were sensitive to imipenem and tigecycline. All (100\%) ESBL negative strains were sensitive to colistin and piperacillin/tazobactam; but $93.9 \%$ and $90.6 \%$ of ESBL positive strains were sensitive to colistin and piperacillin/tazobactam respectively. For cefoxitin sensitivity of ESBL positive and negative E. coli was $85.9 \%$ and $100 \%$ respectively. Sensitivity to amikacin was about $76.5 \%$ and $77 \%$ against ESBL positive and ESBL negative strains respectively. Nitrofurantoin was used in case of urine isolates only where it was almost equally sensitive $(85.2 \%$ vs $84.2 \%$ ) to both of ESBL positive and ESBL negative strains. Sensitivity of ESBL positive organisms to other antibiotics was less; but ESBL negative strains were more sensitive than ESBL positive ones. All ESBL positive organisms were resistant to cephalosporins $\left(1^{\text {st }}\right.$ to $3^{\text {rd }}$ generation) while all ESBL negative organisms were sensitive to cephalosporins.

The scenario of antibiotic sensitivity was similar in case of Klebsiella spp. except that all $(100 \%)$ were sensitive to colistin; $5.6 \%, 19.4 \%$ and $19.4 \%$ of ESBL positive strains were resistant to imipenem, tigecycline and piperacillin/tazobactam respectively. ESBL negative urine isolates showed higher $(65.8 \%)$ sensitivity to nitrofurantoin than ESBL positive ones $(31.6 \%)$. 
Table III: Sensitivity pattern of ESBL positive and ESBL negative E. coli and Klebsiella spp. to different antibiotics (except cephalosporins) $(\mathrm{N}=472)$

\begin{tabular}{|c|c|c|c|c|}
\hline \multirow[b]{2}{*}{ Antibiotics } & \multicolumn{2}{|c|}{ E. coli } & \multicolumn{2}{|c|}{ Klebseilla spp. } \\
\hline & $\begin{array}{c}\text { ESBL +ve } \\
(n=213)\end{array}$ & $\begin{array}{l}\text { ESBL -ve } \\
(n=178)\end{array}$ & $\begin{array}{c}\text { ESBL +ve } \\
\quad(n=36)\end{array}$ & $\begin{array}{c}\text { ESBL -ve } \\
(n=45)\end{array}$ \\
\hline Imipenem & $213(100)$ & $178(100)$ & $34(94.4)$ & $45(100)$ \\
\hline Tigecycline & $213(100)$ & $178(100)$ & $29(80.6)$ & $45(100)$ \\
\hline Colistin & $200(93.9)$ & $178(100)$ & $36(100)$ & $45(100)$ \\
\hline $\begin{array}{l}\text { Piperacillin/ } \\
\text { Tazobactam }\end{array}$ & 193 (90.6) & $178(100)$ & $29(80.6)$ & $45(100)$ \\
\hline Cefoxitin & $183(85.9)$ & $178(100)$ & $31(86.1)$ & $42(93.3)$ \\
\hline Gentamicin & $165(77.5)$ & $168(94.4)$ & $20(55.6)$ & 44 (97.8) \\
\hline Amikacin & $163(76.5)$ & $137(77.0)$ & $30(83.3)$ & 40 (88.9) \\
\hline Doxicycline & $62(29.1)$ & $126(70.8)$ & $00(00)$ & $31(68.9)$ \\
\hline Cotrimoxazole & $53(24.9)$ & $97(54.5)$ & 07 (19.4) & $38(84.4)$ \\
\hline $\begin{array}{l}\text { Amoxycillin/ } \\
\text { Clavulanic acid }\end{array}$ & $42(19.7)$ & $154(86.5)$ & 04 (11.1) & $36(80.0)$ \\
\hline Ciprofloxacin & $24(11.3)$ & $103(57.9)$ & $06(16.7)$ & $42(93.3)$ \\
\hline Nitrofurantoin* & $156(85.2)$ & $144(84.2)$ & $06(31.6)$ & $25(65.8)$ \\
\hline Nalidixic acid* & $08(4.4)$ & $35(20.5)$ & $00(00)$ & 31(81.6) \\
\hline
\end{tabular}

*Urine isolates only; In urine isolates, ESBL +ve E coli 183 , ESBL -ve $E$ coli 171 and ESBL +ve Klebsiella spp.19, ESBL -ve Klebsiella spp. 38

\section{Discussion}

Reporting of ESBL positive organisms with their antibiogram is essential for proper treatment of the patients. In this study the latest scenario of antibiogram and frequency in some health care centres in and around Dhaka city have been presented.

From this study, it became evident that ESBL positive organisms are prevalent throughout the Dhaka city. The overall frequency of ESBL positive organisms was $52.8 \%$ $(n=472)$. The highest frequency of ESBL positive organisms was in Wari Hospital (91.3\%) followed by BIHS Gnereal Hospital IPD, Mirpur (81.4\%) and Foot Care Hospital at Rampura (74.4\%). The lowest frequency of ESBL positive organisms in Banani (32.4\%), the area where most affluent people live, apparently gives a good relationship of economic status, improved environmental sanitation and infection control.
In this study, 472 specimens were tested, of which 249 (52.8\%) were found positive for ESBL organisms. This is consistent with findings of Ahmed et al $(53.4 \%, \mathrm{n}=221)^{1}$ and Sashirekha $(48.9 \%, \mathrm{n}=225)^{16}$. Findings of Dalela $(61.6 \%$, $\mathrm{n}=219)^{17}$, Oliveira et el $(61.1 \%, \mathrm{n}=90)^{18}$ and $\operatorname{Yasmin}^{19}(71.3 \%, \mathrm{n}=300)$ were slightly higher; the findings of Rahman et $\mathrm{al}^{6}$ was slightly lower $(41.4 \%, \mathrm{n}=157)$, but that of Hansen et $\mathrm{al}^{7}(2.4 \%$, $\mathrm{n}=14674$ ) was very low. It is to be noted that in countries where there is good infection control practices and antimicrobial stewardship, the prevalence of ESBL + ve organisms is lower than other countries as was evidenced in a study in 2009 where global prevalence rate was found as follows: Latin America-44\%, Asia/Pacific $-22.4 \%$, Europe-13.3\% and North America$7.5 \%{ }^{20}$ However, prevalence of ESBL positive organisms is increasing throughout the world. ${ }^{4}$

Predominating organism in our study was $E$. coli $(82.8 \%, \mathrm{n}=472)$ and the rest $(17.2 \%)$ was Klebsiella spp.; $54.5 \%$ of E. coli $(\mathrm{n}=391)$ and $44.4 \%$ of Klebsiella spp. $(\mathrm{n}=81)$ were ESBL positive. These findings are consistent with the study of Rahman et $\mathrm{al}^{6}$ and Sashirekha. ${ }^{16}$ In the study of Rahman et $\mathrm{al}^{6}$ predominating organism was E. coli $(54.4 \%, \mathrm{n}=241)$ of which $43.2 \%$ were ESBL positive and $45.6 \%$ were $K$. pneumoniae of which $39.5 \%$ were ESBL positive. In the study of Sashirekha ${ }^{16}$ predominating organism was E. coli $(28 \%$, $\mathrm{n}=325)$ of which $52.8 \%$ were ESBL positive and $25.2 \%$ were $K$. pneumoniae of which $45.1 \%$ were ESBL positive. In the study of Dalela ${ }^{17}$ $73.5 \%$ of E. coli and $58.1 \%$ of $K$. pneumoniae were ESBL positive. In contrast, study of Olveira et al ${ }^{18}$ showed $K$. pneumoniae $(71.1 \%$, n=90) as predominating organism followed by $E$. coli $(24.4 \%) ; 71.9 \%$ of $K$. pneumoniae and $36.4 \%$ of E. coli were ESBL positive. Study of Yasmin $^{19}$ in Bangladesh also showed different scenario where predominating organism was $E$. coli $(52 \%, \mathrm{n}=300)$ followed by Proteus spp. (18.3\%) and Klebsiella spp. (15\%). In her study, Klebsiella spp. (80\%) topped the list as ESBL producers followed by Proteus spp. (72\%) and E. coli $(67.3 \%)$. 
In our study organisms were isolated from two types of specimens - urine and pus. Urine isolates were $87.1 \%$ and pus $12.9 \%$. Out of 411 urine isolates, predominant organism was E. coli $(86.1 \%)$ of which $51.7 \%$ were ESBL positive. Klebsiella spp. was $13.9 \%$ of which $33.3 \%$ were ESBL positive. Our findings are in consistence with that of Yasmin ${ }^{19}$ and Dalela ${ }^{17}$ except that the rate of ESBL positive organisms in urine isolates was higher $(\sim 70-80 \%)$.

In the present study organisms were isolated from 61 pus specimens $-60.7 \%$ were E.coli and $39.3 \%$ were Klebsiella spp. Rate of ESBL positive E. coli and Klebsiella spp. was $81.1 \%$ and $70.8 \%$ respectively. This is consistent with the study of Yasmin ${ }^{19}$ and Dalela ${ }^{17}$ except that the rate of ESBL positive E. coli in the study of Yasmin ${ }^{19}(50 \%, \mathrm{n}=42)$ and rate of ESBL positive Klebsiella spp. in the study of Dalela $(50 \%, \mathrm{n}=20)^{17}$ were lower.

ESBL producing organism is now a global threat ${ }^{1}$ and its treatment has become difficult because of drug resistance over a wide spectrum of $\beta$-lactam and non $\beta$ lactam drugs. In this study, in case of most antibiotics the difference of sensitivity between ESBL positive and ESBL negative organisms was statistically significant.

All ESBL positive organisms (100\%) were found resistant to cephalosporins (except cephamycins as discussed below) and monobactams while all (100\%) ESBL negative organisms were sensitive to these drugs. Other authors showed varying degrees of sensitivity of ESBL producers to cephalosporins. ${ }^{1,16,17}$ It is to be noted that ESBL producers should be taken as resistant to all cephalosporins and monobactams even if these show sensitivity against some of the members. Cefepime is considered as a therapeutic option by some authors, but clinical data show high failure rate. ${ }^{20}$ Some patients with ESBL positive organisms were treated successfully with cephalosporins where organisms appeared sensitive in disc diffusion methods and had very low MIC. 1,16,17,21,22 However, MIC is not done routinely in our department except in cases of multidrug resistance when higher than MIC level of antibiotic is targeted.

Currently, carbapenems are the most effective antibiotic for treatment of infections due to ESBL producing organisms as the outcome is better than treatment with other antibiotics. ${ }^{4,18,22,23}$ This has been true in this study also where all $(100 \%, \mathrm{n}=391) E$ coli strains, both ESBL positive and negative, were sensitive to imipenem. This is consistent with the findings of Sasirekha $(n=225)^{16}$, Yasmin $(n=201)^{19}$ and Paterson ${ }^{23}$ who also found $100 \%$ of isolates sensitive to imipenem. Dalela $(n=215)^{17}$ found $98.5 \% \quad(n=135)$ of ESBL producer and 94\% $(n=219)$ of non-ESBL producers sensitive to imipenem and Ahmed et al ${ }^{1}$ found $98.3 \%$ of ESBL producer E. coli sensitive to imipenem. However, $5.6 \%$ of ESBL positive Klebsiella spp. in our study showed resistance to imipenem which is similar to the study of Hawser et $\mathrm{al}^{24}$ where $10 \%$ of ESBL positive Klebsiella spp. were resistant to imipenem; but it contrasts with the study of Sasirekha ${ }^{16}$, Yasmin ${ }^{19}$ and Paterson $^{23}$ who found all ESBL producing Klebsiella spp. sensitive to imipenem. Imipenem resistance of ESBL positive Klebsiella spp. is increasing all over the world day by day. ${ }^{22}$

In this study all isolates except ESBL positive Klebsiella spp. were found sensitive to tigecycline; $80.6 \%$ of ESBL +ve Klebsiella spp. were sensitive to tigecycline. In other studies, it was found that tigecycline had excellent in vitro sensitivity but data reflecting clinical outcome is lacking. ${ }^{20,23,25}$ It was also reported that there was increased mortality rate in tigecycline-treated patients than in patients treated by other antibiotics. ${ }^{20}$ FDA warned health professionals and their medical care organizations about the increased risk of death when intravenous tigecycline is used. They suggested to reserve tigecycline for use only in situations when alternative treatments are not suitable. ${ }^{26}$

About $94 \%$ of ESBL positive E. coli were found sensitive to colistin in this study. All ESBL negative $E$. coli and all Klebsiella spp. were sensitive to colistin. Other studies also demonstrated its efficacy in treating multidrug resistant organisms including ESBL producers. ${ }^{20,23}$ Although recent studies have shown that it has acceptable effectiveness and has been used to treat infections due to multiresistant Gram-negative organisms, its use should be reserved as a last resort mainly for ESBL producing bacteria that are also resistant to aminoglycosides and carbapenems. ${ }^{25}$

In this study, $<20 \%$ ESBL producers and $>80 \%$ nonESBL producers (both E. coli and Klebsiella) were sensitive to amoxycillin/clavulanic acid. In the study of Dalela $^{17}, 15.6 \%$ of ESBL producers and $23.8 \%$ of non- 
ESBL producers were sensitive to amoxycillin/ clavulanic acid.

In our study all (100\%) ESBL negative E. coli and Klebsiella spp. were found sensitive to piperacillin/tazobactam whereas $90.6 \%$ of ESBL positive E. coli and $80.6 \%$ of ESBL positive Klebsiella spp. were sensitive to piperacillin/tazobactam. Dalela ${ }^{17}$ found $65.5 \%$ of non-ESBL producers and $72.6 \%$ of ESBL producers sensitive to piperacillin/tazobactam. Sasirekha ${ }^{16}$ found about $70 \%$ of non-ESBL producers and $60 \%$ of ESBL producers sensitive to piperacillin/tazobactam. Other studies showed that clinical experience with $\beta$-lactam/ $\beta$-lactamase inhibitor combinations in the treatment of severe infections due to ESBL producers is limited and showed mixed results; but it may be an option of treatment for infections like urinary tract infections (UTI) caused by susceptible ESBLs. ${ }^{20-23}$

The cephamycins (e.g. cefoxitin) which are also $2^{\text {nd }}$ generation cephalosporins demonstrate markedly greater resistance to microbial degradation by $\beta$ lactamases than is normally displayed by the cephalosporins. $^{22}$ In our study, $85.9 \%$ of ESBL positive and $100 \%$ of ESBL negative E. coli and $86.1 \%$ of ESBL positive and $93.3 \%$ of ESBL negative Klebsiella were sensitive to cefoxitin. However, clinical data regarding its use is scarce and clinical failure has been documented due to co-resistance. ${ }^{4,20}$

In this study about $76.5 \%$ of ESBL positive and $77 \%$ ESBL negative strains of $E$. coli showed sensitivity against amikacin and comparatively it was higher in ESBL positive $(83.3 \%)$ and ESBL negative $(88.9 \%)$ Klebsiella spp. These findings are consistent with that of Yasmin ${ }^{19}$ who found $83 \%$ of E. coli and $81 \%$ of Klebsiella spp. sensitive to amikacin. Dalela ${ }^{17}$ found $64.5 \%$ of ESBL producers and $46.4 \%$ of non-ESBL producer organisms sensitive to amikacin. About $78 \%$ ESBL producers and $60 \%$ of non-ESBL producers were susceptible to amikacin in the study of Ahmed et al. ${ }^{1}$ But in contrast to these findings less sensitivity was observed in the study of Sasirekha ${ }^{16}$ where only $30 \%$ of ESBL producers and about $40 \%$ of non-ESBL producers were sensitive to amikacin.

In our study, $77.5 \%$ of ESBL positive E. coli and 55.6\% of ESBL positive Klebsiella spp. were sensitive to gentamicin whereas $94.4 \%$ of ESBL negative E. coli and $97.8 \%$ of ESBL negative Klebsiella spp. were sensitive to this drug. In the study of Ahmed et al ${ }^{1}$ $34.8 \%$ of ESBL positive and $21.5 \%$ of ESBL negative E. coli were sensitive to gentamicin. However, aminoglycosides may be potentially useful in the treatment of complicated urinary tract infections due to ESBL-producing organisms; but these are not recommended as routine monotherapy for severe infections at other sites. ${ }^{20,23}$

Nitrofurantoin was used in case of urine isolates only; both ESBL positive and ESBL negative E. coli were found almost equally sensitive ( $85 \%)$. But Klebsiella spp. showed different figure $-31.6 \%$ of ESBL positive Klebsiella spp. and $65.8 \%$ of ESBL negative strains were sensitive to nitrofurantoin. Our findings are almost similar to that of Yasmin ${ }^{19}$ in case of E. coli (about $96 \%$ sensitive) but differed in case of Klebsiella spp. (about $95 \%$ sensitive). Ahmed et $\mathrm{al}^{1}$ found $54 \%$ cases sensitive in case of both ESBL positive and negative E. coli. Sasirekha ${ }^{16}$ found about $40 \%$ cases sensitive in case of both ESBL producer and non-producer organisms.

Sensitivity of ESBL positive organisms to other antibiotics was very low (doxycycline $29.1 \%$ and zero, cotrimoxazole $24.9 \%$ and $19.4 \%$ and ciprofloxacin $11.3 \%$ and $16.7 \%$ for E.coli and Klebsiella spp. respectively). But sensitivity of ESBL negative strains were significantly higher than ESBL positive organisms $(p<0.05)$. These findings are consistent with that of Yasmin $^{19}$, Dalela ${ }^{17}$, Ahmed et $\mathrm{al}^{1}$ and Sasirekha ${ }^{16}$.

While reporting the culture and sensitivity tests, the ESBL positive organisms should be pointed out with comment like this - "The organisms are ESBL positive and resistant to penicillins, cephalosporins and monobactams". In this study imipenem was found as the most effective drug for treatment of ESBL positive as well as ESBL negative organisms followed by colistin, tigecycline, piperacillin/tazobactam. However, these drugs should be kept reserved and used only when other sensitive drugs are not available so that emergence of resistance against these drugs is deferred. While selecting antibiotic against ESBL positive organisms, it should be remembered that this group of organisms are relatively less sensitive than ESBL negative organisms. Also in cases of ESBL positive organisms, it seems prudent to avoid $\beta$-lactam antibiotics as far as possible. 


\section{References}

1. Ahmed K, Thokar MA, Toboli AS, Fomda BA, Bashir G, Maroof $\mathrm{P}$ et al. Extended spectrum $\beta$-lactamase mediated resistance in Escherichia coli in a tertiary care hospital in Kashmir, India. Afr. J. Microbiol. Res. 2010; 4(24): 2720-2728.

2. Chaudhury U, Aggarwal R. Extended spectrum $\beta$-lactamases (ESBL) - an emerging threat to clinical tharapies. Ind J Med Microbiol 2004; 22(2): 75-80.

3. Knothe H, Shah P, Kremery V, Anta IM, Mitsuhashi S. Transferable resistance to cefotaxime, cefoxitin, cefomandole and cefuroxime in clinical isolates of Klebsiella pneumonia and Serratia marsescens. Infection 1983; 11(6): 315-317.

4. Rupp ME, Fey PD. Extended spectrum $\beta$-lactamase (ESBL)producing Enterobacteriaceae. Drugs 2003; 63(4): 353-365.

5. Jacoby JA. Extended spectrum $\beta$-lactamases and other enzymes providing resistance to oxyimino $\beta$-lactams. Infect Dis Clin North Am 1997; 11: 875-887.

6. Rahman MM, Haq JA, Hossain MA, Sultana R, Islam F, Islam AHMS. Prevalence of extended spectrum $\beta$-lactamaseproducing Escherichia coli and Klebsiella pneumonia in an urban hospital in Dhaka, Bangladesh. International Journal of Antimicrobial Agents 2004; 24: 508-510.

7. Hansen DS, Schumacher H, Hansen F, Stegger M, Hertz FB, Schonnning K et al. Extended spectrum $\beta$-lactamase (ESBL) in Danis clinical isolates of Escherichia coli and Klebsiella pneumonia: prevalence, $\beta$-lactamase distribution, phylogroups and co-resistance. Scan J Infect Dis 2012; 44(3): 174-181.

8. Lahey. $\beta$-Lactamase classification and amino acid sequences for TEM, SHV and OXA extended-spectrum and inhibitor resistant enzymes. Available at: http://www.laheyorg/ studies/ Accessed June 2013.

9. Fennell J, Vellinga A, Hanahoe B, Morris D, Boyle F, Higgins $\mathrm{F}$ et al. Increasing prevalence of ESBL production among Irish clinical Enterobacteriaceae from 2004 to 2008: an observational study. BMC Infectious Diseases 2012; 12: 116-123.

10. Dhillon RHP, Clark J. ESBLs: a clear and present danger? Critical Care Research Practice 2012: 1-11. Article ID 625170 .

11. Nataro JP, Bopp CA, Fields PI, Kaper JB, Strockbine NA. Escherichia, Shigella and Salmonella. In: Versalovic J, Carrol KC, Funke G, Jorgensen JH, Landry ML, Warnock DW (eds). Manual of clinical microbiology (Vol 1). $10^{\text {th }}$ edn. Washington DC: American Society for Microbiology Press, 2011: 603-624.
12. Abbott SL. Klebsiella, Enterobacter, Citrobacter, Serratia, Plesiomonas and others. In: Versalovic J, Carrol KC, Funke G, Jorgensen JH, Landry ML, Warnock DW (eds). Manual of clinical microbiology (Vol 1). $10^{\text {th }}$ edn. Washington DC: American Society for Microbiology Press, 2011: 639-657.

13. CLSI. Performance standard for antimicrobial susceptibility testing; $22^{\text {nd }}$ information supplement. M100-S22, 2012; 32(3): 20-60.

14. NCCLS. Performance standards for antimicrobial disk susceptibility tests. $6^{\text {th }}$ edn. Approved standard 1997 January. M2-A6; 17(1): 5-17.

15. Jarlier V, Nicolas MH, Fournier G, Philoppon A. Extended broad-spectrum beta-lactamases conferring transferrable resistance to newer beta-lactam agents in Enterobacteriaceae: hospital prevalence and susceptibility pattern. Rev Infect Dis 1988; 10(4): 867-878.

16. Sasirekha B. Prevalence of ESBL, AMPC B-Lactamases and MRSA among uropathogens and its antibiogram. EXCLI Journal 2013; 12: 81-88.

17. Dalela G. Prevalence of extended spectrum Beta lactamase (ESBL) producers among Gram negative bacilli from various clinical isolates in a tertiary care hospital at Jhalawar, Rajasthan, India. Journal of Clinical and Diagnostic Research 2012; 6(2): 182-187.

18. Oliveira CFD, Salla A, Lara VM, Rieger A, Horta JA, Alves SH. Prevalence of extended spectrum beta-lactamasesproducing microorganisms in nosocomial patients and molecular characterization of the SHV type isolates. Brazilian Journal of Microbiology 2010; 41: 278-282.

19. Yasmin T. Prevalence of ESBL among Esch. coli and Klebsiella spp. in a tertiary care hospital and molecular detection of important ESBL producing genes by multiplex PCR [M.Phil thesis]. Mymensingh: Mymensingh Medical College; 2012.

20. Dhillon RHP, Clark J. ESBLs: a clear and present danger? Critical Care Research and Practice 2012: 1-11. Article ID 625170.

21. Daoust DR, Onishi HR, Wallick H, Hendin D, Stapley EO. Cephamycins, a new family of $\beta$-lactam antibiotics: antibacterial activity and resistance to $\beta$-lactamase degradation. Antimicrobial Agents and Chemotherapy 1973; 3(2): 254-261.

22. Nguyen HM, Shier KL, Graber CJ. Determining a clinical framework for use of cefepime and $\beta$-lactam/ $\beta$-lactamase inhibitors in the treatment of infections caused by extendedspectrum- $\beta$-lactamase-producing Enterobacteriaceae. J Antimicrob Chemother 2014; 69(4): 871-880. 
23. Paterson DL. Infections due to extended spectrum betalactamase (esbl)-producing microorganisms: an epidemiological and therapeutic challenge. Enferm Infect Microbiol Clin 2007; 25(Suppl 2): 60-63.

24. Hawser S, Hoban DH, Bouchillon S, Badal R, Carmeli Y, Hawkey P. Antimicrobial susceptibility of intra-abdominal Gram-negative bacilli from Europe: SMART Europe 2008. Eur J Clin Microbiol Infect Dis 2011; 30: 173-179.
25. Pallett A, Hand K. Complicated urinary tract infections: practical solutions for the treatment of multiresistant Gramnegative bacteria. J Antimicrob Chemother 2010; 65(Suppl 3): $25-33$.

26. FDA. Tygacil (tigecycline): drug safety communicationincreased risk of death. Available at: http://www. fda.gov/Safety/MedWatch/SafetyInformation/SafetyAlerts for Human Medical Products/ucm370170.htm. Accessed September 2013. 